\title{
Rate Distribution Modeling for Structured Heterogeneous Populations*
}

\author{
Ben G. Fitzpatrick \\ Center for Research in Scientific Computation \\ and \\ Department of Mathematics \\ North Carolina State University \\ Raleigh, NC 27695-8205
}

* Research supported in part under AFOSR contract F49620-93-1-0153. 


\begin{abstract}
We propose a modeling strategy for structured populations, in which individuals are not necessarily identical. The heterogeneity is obtained by modeling the population as comprising homogeneous subpopulations. By using a vector measure, we combine the subpopulations with an abstract integral to obtain the density of the population. We show that this approach leads to a semigroup formulation of the dynamics in a space of vector measures, and we develop some estimation methods for determining the initial structure from observed data.
\end{abstract}

\title{
1. Introduction
}

Many biological applications of dynamical systems and control theory involve populations that are structured in terms of size, age, or spatial distribution. From predicting future populations to developing optimal harvesting strategies, models provide important information for biologist. Thus, it is crucial to develop models that predict population behavior in an accurate manner. This paper focuses on a particular aspect of model improvement, that of incorporating individual-based information into aggregate, population-wide models.

The original motivation for this work involved certain behavior exhibited by observed data for a size structured population of mosquitofish, behavior which is inconsistent with the commonly used Sinko-Streifer model:

$$
v_{t}+(g v)_{x}=-\mu v, \quad x_{0}<x<x_{1}, t>0
$$

Here $v=v(t, x)$ denotes the density of indiviuals of size $x$ at time $t, g=g(t, x)$ denotes individual growth rate, and $\mu=\mu(t, x)$ is the mortality rate. The observed data, as discussed in [BBKW, BF], exhibits a dispersion in size as time increases, and in some cases unimodal initial densities develop into bimodal densities at later time: the Sinko-Streifer 
model does not predict these phenomena without biologically unrealistic assumptions on the parameters.

In order to improve model predictions, various researchers (see $[\mathrm{BBKW}, \mathrm{BF}]$ ) have attempted to examine the model on the individual level. In general, individuals do not have identical growth rates (a basic assumption for the Sinko-Streifer model). To introduce individual variations, we model the population as being composed of homogeneous subpopulations, with each subpopulation obeying a Sinko-Striefer law having different parameters. The approach in $[\mathrm{BBKW}, \mathrm{BF}]$ involves subpoulation densities $v(t, x ; g)$, with the growth rate parameter $g$ differentiating the subpopulations. The subpopulations are then be combined by integrating the densities with respect to a measure on the space of parameters:

$$
u(t, x)=\int_{G} v(t, x ; g) d P(g)
$$

The measure $P$, called the growth rate distribution, represents the proportion of individuals having a given growth rate.

In this paper, we consider the more general problem of a population whose dynamics are modeled with a parameter-dependent $C_{0}$ semigroup $T(t ; q)$ on a Hilbert space $X$. These operators typically arise as solution operators for a differential equation such as the Sinko-Streifer equation given above. This general approach allows us to consider several different kinds of models from many differential equations - age, size, spatial structure models, or a combination. Within the context of population models, the parameter $q$ typically comprises several individual rate parameters, such as growth, mortality, and fecundity. The rate distribution idea then is to model the population as a combination of subpopulations which are modeled with the original semigroup dynamics $T(t ; q)$. The question then becomes how to build the whole population from the subpopulations. 
Suppose for the moment that the number of subpopulations is finite. Then, we denote the densities by $v\left(t, x ; q_{i}\right)=T\left(t, q_{i}\right) \varphi_{i}(x)$, for $1 \leq i \leq n$. Then the density $u(t, x)$ of the whole population is given by

$$
u(t, x)=\sum_{i=1}^{n} T\left(t, q_{i}\right) \varphi_{i}(x)
$$

In $[\mathrm{BF}]$, the initial densities were assumed to satisfy

$$
\varphi_{i}=p_{i} \varphi,
$$

for some $\varphi \in X$, and some collection $\left\{p_{i}\right\}$ with $p_{i} \geq 0$, and $\sum p_{i}=1$. Then the population density can be given in the form

$$
u(t, x)=\sum_{i=1}^{n} T\left(t, q_{i}\right) \varphi(x) p_{i}=\int_{Q} T(t ; q) \varphi(x) d P(q),
$$

where $P$ is the discrete probability measure on $Q$ with support $\left\{q_{i}\right\}$ and weights $\left\{p_{i}\right\}$. The integral form is generalized naturally to the case mentioned above in which $P$ is any probability measure on $Q$, and the estimation theory of $[\mathrm{BF}]$ generalizes in a straightforward manner for this problem.

To generalize this model further, we relax (1.2), so that the subpopulations may have different initial structure as well as distinct rate parameters. Thus we are led to a generalization of the form

$$
u(t)=\int_{Q} T(t ; q) \varphi(q) d P(q),
$$

where the $X$ valued function $\varphi(q)$ gives the parameter dependent initial structure. A further step is to write

$$
u(t)=\int_{Q} T(t ; q) d \mathbf{m}(q),
$$

where $\mathbf{m}$ is a vector-valued measure on $Q$ (taking values in $X$ ). Intuitively, we have $\mathbf{m}(d q)=\varphi(q) P(d q)$. Also, when $X=L^{2}$, say, $\int_{a}^{b} \mathbf{m}(A)(x) d x$ denotes the number of individuals whose structure variable $x$ is between $a$ and $b$, and whose parameter $q$ lies 
in the set $A \subset Q$. Note that formula (1.4) above involves integrating an operator valued function with respect to a vector measure. There are some subtle questions of measurability in both (1.3) and (1.4) that must be resolved from properties of the original semigroup model (see $[\mathrm{F}]$ ).

The advantage to using the form (1.4) over (1.3) is not in a greater level of generality, for under rather general conditions a vector measure can be expressed in terms of a vector valued density and a scalar measure. However, as we shall see below, the vector measure approach leads directly to a semigroup formulation of the dynamics in a vector measure space, and hence we may view the state as possessing not only the original (size, age, or spatial) structure but also the individual (rate) structure.

We shall consider the population state at time $t$ to be $\mathbf{m}_{t}$, which can be given in mild form at

$$
\mathbf{m}_{t}=\mathcal{T}(t) \mathbf{m}_{0}+\int_{0}^{t} \mathcal{T}(t-s) \mathbf{f}(s) d s,
$$

where $\mathbf{f}$ is a "forcing function" modeling changes in parameter structure due to external environmental changes. Below we shall derive the semigroup $\mathcal{T}$ from $T(t ; q)$ : the idea is that $\mathcal{T}(t) \mathbf{m}_{0}(d q)=T(t ; q) \varphi(q) \mu(d q)$, where we have $\mathbf{m}_{0}(d q)=\varphi(q) \mu(d q)$.

The inverse problem is to estimate the measure $\mathbf{m}_{0}$ and the forcing function $\mathbf{f}$ from observations of the population. In size structured populations, one typically obtains "histogram" data which represents numbers of individuals whose size lies in given "size bins." We assume that the observations are of the form $\left\{\widehat{u}\left(t_{i}\right): 1 \leq i \leq n\right\} \subset Z$, where the data space $Z$ is a Hilbert space, and we use the least squares criterion

$$
J\left(\mathbf{m}_{0}, \mathbf{f}\right)=\sum_{k=1}^{n}\left\|\widehat{u}\left(t_{k}\right)-\mathcal{C} \mathbf{m}_{t}(Q)\right\|_{Z}^{2},
$$

where $\|\cdot\|_{Z}$ denotes the norm in $Z$, and where $\mathcal{C}: X \rightarrow Z$ is the observation mapping (e.g., a projection onto the step functions in the histogram case). Computation of solutions to this inverse problem requires several approximations - for the semigroup, the vector 
integral in (1.5), and the vector measure itself. It does, however, provide an advantage over identification of the parameter $q$ : the least squares problem for the measure is a linear least squares problem. A very similar idea, relaxed control, has been used in control theory for quite some time (see, e.g. [W]).

A computational advantage in this approach is that the problem contains a high level of parallelism. Different subpopulations can be simulated independently; hence, parallel computing platforms can be used very efficiently.

This paper is organized as follows. In Section 2, we discuss the functional analytic tools necessary to put (1.5) on a rigorous foundation. Section 3 contains the approximation methods for inverse problems, and some remarks on future work are contained in Section 4. 


\section{Semigroup Formulation of the Model.}

Our goal in this section is to extend the dynamics of a population comprising identical individuals to a heterogeneous population, distributed not only in its original structure but also in its rate structure. We begin with the original dynamics,

$$
\dot{v}(t ; q)=A(q) v(t ; q),
$$

where $A$ is the infinitestimal generator of a $C_{0}$ semigroup $T(t ; q)$ on a Hilbert space $X$. In the extension we are seeking, the solutions $v(t ; q)$ represent the subpopulation density having parameter $q$. Of course, when we refer to this function of $q$ as a density, we need a measure with respect to which $v$ is a density. We also need an extended state space which incorporates the additional structure.

One natural approach to a state space formulation is to take the initial population to be an $X$-valued measure. The space $\mathcal{M}$ of regular, countably additive $X$-valued Borel measures of bounded variation on $Q$ forms a Banach space which can be identified with $C(Q, X)^{*}$. We include here some definitions and notation which will be very useful below. The interested reader may consult $[\mathrm{DU}]$ or $[\mathrm{D}]$ for the details of vector measures. First, we recall that a countably additive vector measure (or more concisely, a vector measure) is a function from a $\sigma$-algebra $\mathcal{F}$ of sets in $Q$ to $X$ satisfying

$$
\mathbf{m}\left(\bigcup_{i=1}^{\infty} E_{i}\right)=\sum_{i=1}^{\infty} \mathbf{m}\left(E_{i}\right)
$$

for every collection $\left\{E_{i}\right\} \subset \mathcal{F}$ of disjoint sets. The limit in the infinite sum holds in the $X$ norm sense. We define the variation of $\mathbf{m},|\mathbf{m}|$, by

$$
|\mathbf{m}|(E)=\sup _{\pi \in \Pi} \sum_{E_{i} \in \pi}\left\|\mathbf{m}\left(E_{i}\right)\right\|_{X},
$$

where $\Pi$ is the collection of finite partitions of $E$. If $|\mathbf{m}|(Q)<\infty$, we say that $\mathbf{m}$ is of bounded variation. The semivariation is defined by

$$
\|\mathbf{m}\|(E)=\sup \left\{|\langle x, \mathbf{m}(E)\rangle|: x \in X,\|x\|_{X}=1\right\},
$$


where $\langle\cdot, \cdot\rangle$ denotes the inner product in $X$. We say that $\mathbf{m}$ is of bounded semivariation if $\|\mathbf{m}\|(Q)<\infty$. A vector measure is of bounded semivariation if and only if its range is bounded in $X$ (p. 5 of [DU]) so that we refer to measures of bounded semivariation simply as bounded measures. Unless otherwise stated, integrals of vector valued functions are taken to be Bochner integrals (see [DU, Chapter 2]).

Measures of bounded variation can also be expressed as $\mathbf{m}(d q)=\varphi(q) \mu(d q)$, where $\varphi \in L^{1}(Q, \mu ; X)$ and $\mu$ is a positive real-valued measure on $Q$, such that $0 \leq \mu(A) \leq$ $\|\mathbf{m}\|(A)$, for each measurable $A$. This fact follows from a theorem of Bartle, Dunford, and Schwarz and the fact that Hilbert spaces have the Radon-Nikodym property (see [DU, pp. 14,61 , and 100]).

Another state space possibility is given by $C(Q ; X) \times M$, where $M\left(=C(Q)^{*}\right)$ denotes the finite real-valued Borel measures on $Q$, for $Q$ a compact separable metric space. This approach has been successfully employed in [BKW] within the context of model development.

Here we shall focus on the vector measure approach, which has proven effective in inverse problems (see $[\mathrm{F}]$ and the following sections). We begin by extending the original semigroup to the vector measure space. The following result is the first necessary step.

Lemma 2.1. Suppose that $X$ is a Hilbert space and that $T(t ; q)$ is a semigroup on $X$ that is strongly jointly continuous on $[0, \infty) \times Q$, where $Q$ is a compact, separable metric space. Suppose that $\mu$ is a finite regular nonnegative measure on (the Borel sets of) $Q$ and that $\varphi \in L^{1}(Q, \mu ; X)$. Then, for each $t$, the map $q \mapsto T(t, q) \varphi(q)$ is in $L^{1}(Q, \mu ; X)$, and the vector measure $\mathbf{n}$ given by $\mathbf{n}(A)=\int_{A} T(t, q) \varphi(q) \mu(d q)$ is in $\mathcal{M}$.

Proof. First, we note that if the above map is measurable, then we have that

$$
\begin{aligned}
\int_{Q}\|T(t ; q) \varphi(q)\|_{X} \mu(d q) & \leq \int_{Q}\|T(t ; q)\|\|\varphi(q)\|_{X} \mu(d q) \\
& \leq M e^{\omega t} \int_{Q}\|\varphi(q)\|_{X} \mu(d q)<\infty
\end{aligned}
$$


since $T$ is jointly strongly continous (see $[D U, F]$ ). Thus, to prove that the above map is $L^{1}$, it remains to show measurability.

We recall that an $X$-valued function is measurable if and only if it is the ( $\mu$-a.e.) pointwise strong limit of simple functions (see [DU]). Given that $\varphi \in L^{1}(Q, \mu ; X)$, we may choose a sequence of simple functions $\varphi^{n} \rightarrow \varphi$ strongly, for $\mu$-a.e. $q \in Q$. Next, for each $n$, we choose a finite collection of balls of radius $1 / n, B\left(q_{i}^{n}, 1 / n\right)$, for $1 \leq i \leq K_{n}$ that cover $Q$. We redefine these balls in order to make them disjoint, by setting $B_{1}^{n}=B\left(q_{1}^{n}, 1 / n\right)$, and letting

$$
B_{k}^{n}=B\left(q_{k}^{n}, 1 / n\right) \cap\left(B_{1}^{n}\right)^{c} \cap \ldots\left(B_{K_{n}}^{n}\right)^{c} .
$$

We then set

$$
\psi^{n}(q)=\sum_{k=1}^{K_{n}} \sum_{i=1}^{N_{n}} T\left(t, q_{k}^{n}\right) \phi_{i}^{n} \chi_{A_{i}^{n} \cap B_{k}^{n}},
$$

where $\varphi=\sum_{i=1}^{N_{n}} \phi_{i}^{n} \chi_{A_{i}^{n}}$. Clearly, $\psi^{n}$ is a simple function. Moreover, if $q_{0}$ is a point for which $\varphi^{n}\left(q_{0}\right) \rightarrow \varphi(q)$, then there is a sequence of sets $A_{i_{n}}^{n} \cap B_{k_{n}}^{n}$ that contain $q_{0}$ and whose radii are tending to 0 . By the strong continuity of $T$, together with the convergence of $\varphi^{n}$, we have that $T\left(t ; q_{k_{n}}^{n}\right) \varphi^{n}\left(q^{0}\right) \rightarrow T\left(t ; q_{0}\right) \varphi\left(q_{0}\right)$, as desired.

For the last claim, we have that $\mathbf{n}(A)=\int_{A} T(t, q) \varphi(q) \mu(d q)$ is a countably additive, bounded variation vector measure (from [DU, p. 46]) and that in fact

$$
|\mathbf{n}|(A)=\int_{A}\|T(t, q) \varphi(q)\|_{X} \mu(d q)
$$

To prove regularity of $\mathbf{n}$, we must show that for every measurable set $A$ and for every $\varepsilon>0$, there exist sets $K$, compact, and $O$, open satisfying $K \subset A \subset O$, and $|\mathbf{n}|(O-K)<\varepsilon$.

Note that if $\varepsilon>0$, since $q \mapsto T(t, q) \varphi(q)$ is in $L^{1}(Q, \mu ; X)$, there exists $\delta>0$ such that if $B$ is measurable and $\mu(B)<\delta$, then $|\mathbf{n}|(B)<\varepsilon$. The regularity of $\mu$ then provides the result.

Using this lemma, we define the operators $\mathcal{T}(t)$ on $\mathcal{M}$ by setting

$$
\mathcal{T}(t) \mathbf{m}(d q)=T(t ; q) \varphi(q) \mu(d q)
$$


To prove that this definition gives us a semigroup on $\mathcal{M}$, we must first show that $\mathcal{T}(t)$ is well defined. That is, suppose $d \mathbf{m}=\varphi d \mu=\psi d \nu$. (In fact, many such distinct representations exist: see [DU, p. 269, Corollary 3]). We must show that both representations lead to the same linear operator; i.e.,

$$
T(t ; q) \varphi(q) \mu(d q)=T(t ; q) \psi(q) \nu(d q) .
$$

Toward that end, we take $B_{k}^{n}$ and $q_{k}^{N}$ as in the above proof, and we define a sequence of operators

$$
T^{n}(t ; q) x=\sum_{k=1}^{K_{n}} \chi_{B_{k}^{n}}(q) T\left(t ; q_{k}^{N}\right) x,
$$

which converges strongly to $T(t ; q)$. In a manner similar to $(2.2)$, we set

$$
\mathbf{n}_{1}^{n}(A)=\sum_{k=1}^{K_{n}} \int_{A \cap B_{k}^{n}} T\left(t ; q_{k}^{N}\right) \varphi \mu(d q)=\sum_{k=1}^{K_{n}} T\left(t ; q_{k}^{N}\right) \int_{A \cap B_{k}^{n}} \varphi \mu(d q),
$$

and

$$
\mathbf{n}_{2}^{n}(A)=\sum_{k=1}^{K_{n}} \int_{A \cap B_{k}^{n}} T\left(t ; q_{k}^{N}\right) \psi \nu(d q)=\sum_{k=1}^{K_{n}} T\left(t ; q_{k}^{N}\right) \int_{A \cap B_{k}^{n}} \psi \nu(d q) .
$$

From the hypothesis on $\varphi, \psi, \mu$, and $\nu$, we see that $\mathbf{n}_{1}^{n}=\mathbf{n}_{2}^{n}$. Moreover, by the dominated convergence theorem, we have that $\mathbf{n}_{i}^{n}(A) \rightarrow \mathbf{n}_{i}(A)$ for $i=1,2$, where

$$
\mathbf{n}_{1}(A)=\int_{A} T(t, q) \varphi(q) \mu(d q)
$$

and $\mathbf{n}_{2}$ is similarly defined. Since $\mathbf{n}_{1}^{n}=\mathbf{n}_{2}^{n}$, we must have $\mathbf{n}_{1}=\mathbf{n}_{2}$, which implies that $\mathcal{T}(t)$ is well defined.

We also note that since

$$
|\mathbf{n}|(A)=\int_{A}\|T(t, q) \varphi(q)\|_{X} \mu(d q) \leq M e^{\omega t}|\mathbf{m}|(A),
$$

$\mathcal{T}(t)$ is a bounded linear operator on $\mathcal{M}$. Using these facts we have the following.

Theorem 2.2. Under the hypotheses of Lemma 2.1, we have that $\mathcal{T}(t), t \geq 0$ is a strongly continuous semigroup of linear operators on $\mathcal{M}$. 
Proof. The semigroup property is easily seen to follow from (2.3), as is the fact that $\mathcal{T}(0)=I$. We must now argue strong continuity. Note that

$$
|\mathcal{T}(t) \mathbf{m}-\mathbf{m}|(Q)=\int_{Q}\|T(t, q) \varphi(q)-\varphi(q)\|_{X} \mu(d q)
$$

which goes to 0 as $t$ goes to 0 , by the dominated convergence theorem. Thus strong continuity is proved.

From this theorem we obtain a model for dynamics in the vector measure state space, $\mathcal{M}$, through the mild form given in (1.5):

$$
\mathbf{m}_{t}=\mathcal{T}(t) \mathbf{m}_{0}+\int_{0}^{t} \mathcal{T}(t-s) \mathbf{f}(s) d s,
$$

where $\mathbf{f} \in L^{1}\left(\left[0, t_{f}\right] ; \mathcal{M}\right)$. The state vector now is $\mathbf{m}_{t}$ which contains structure in both the parameter space and the original space $X$. Moreover, the mild form also contains a forcing function which can be used to model externally induced parameter changes. Having developed a semigroup formulation for the extended dynamics, we now turn to inverse problems. 


\section{Inverse Problems for the Rate Distribution Model.}

It is typically the case in a population biology application that the individual rate constants cannot be measured directly. Thus, in population wide observations, one would be unlikely to have access to measurements of parameter variation in the population. On the other hand, size and spatial structure are observable in many cases. Thus, we are confronted with an inverse problem in which the observations $\hat{u}\left(t_{i}\right) \in Z$ correspond to $\mathcal{C}\left(\int_{Q} T\left(t_{i} ; q\right) \varphi(q) \mu(d q)\right)$, where $\mathcal{C}: X \rightarrow Z$ is an observation operator and the data space $Z$ is a separable Hilbert space. In many population biology examples, the data are histograms giving the number of individuals whose size (age, position) lies in particular intervals. In such a case $Z$ is finite dimensional and $\mathcal{C}$ is an integral "averaging" operator. The integral in $q$ denotes the summing of the population over the parameter space: we observe the total population, structured only in $X$. We thus pose the inverse problem as the determination of $\mathbf{m}$ from such measurements. We shall use the least squares approach, in which we seek to minimize

$$
J(\mathbf{m})=\sum_{i=1}^{n}\left\|\mathcal{C} \mathcal{T}\left(t_{i}\right) \mathbf{m}(Q)-\hat{u}\left(t_{i}\right)\right\|_{Z},
$$

which, we note, is an infinite dimensional linear least squares problem.

A possible approach to the minimization is through standard linear least squares theory and the normal equations $B^{*} B \mathbf{m}=\hat{u}$, where $B=\left(\mathcal{T}\left(t_{1}\right), \ldots, \mathcal{T}\left(t_{n}\right)\right)^{T} \in \mathcal{L}\left(X^{n}\right)$, and $\hat{u}=\left(\hat{u}\left(t_{1}\right), \ldots, \hat{u}\left(t_{n}\right)\right)^{T} \in X^{n}$. We do not have at this point any general conditions on the original semigroup under which the normal equations have a unique solution. Hence, we take here a different approach.

We treat the problem as a constrained minimization of $J$ over a set $\mathcal{M}_{a d}$ of admissible parameters. Since the unit ball in $C(Q, X)^{*}$ is weak-* compact (by Alaoglu's theorem), we take $\mathcal{M}_{\text {ad }} \subset \mathcal{M}$ to be the measures with variation bounded by $M$, which is a fixed positive number. Under the assumption that the adjoint semigroup, $T^{*}(t ; q)$, is strongly continuous 
in $t$ and $q$, we have that $\mathbf{m}^{N} \rightarrow \mathbf{m}$ weak- ${ }^{*}$ implies that $\mathcal{T}(t) \mathbf{m}^{N}(Q) \rightarrow \mathcal{T}(t) \mathbf{m}(Q)$ weakly in $X$, for

$$
\begin{aligned}
\left\langle x, \int_{Q} T(t ; q) \varphi(q) \mu(d q)\right\rangle & =\int_{Q}\langle x, T(t ; q) \varphi(q)\rangle \mu(d q) \\
& =\int_{Q}\left\langle T^{*}(t ; q) x, \varphi(q)\right\rangle \mu(d q) .
\end{aligned}
$$

Since $T^{*}$ is strongly continuous, $q \mapsto T^{*}(t, q) x \in C(Q, X)$, and the weak convergence becomes apparent. From this convergence we obtain the following.

Theorem 3.1. Suppose that $\mathcal{C} \in \mathcal{L}(X, Z)$ is compact. Under the assumptions of Theorem 2.2 , together with strong continuity of the adjoint semigroup $T^{*}(t ; q)$, the functional $J$ is continuous with respect to the weak-* topology on $\mathcal{M}_{a d}$; hence, $J$ attains a minimum over $\mathcal{M}_{\text {ad }}$.

Proof. First, note that the weak- ${ }^{*}$ topology on $\mathcal{M}_{a d}$ and the weak topology of $\mathcal{T}(t)\left(\mathcal{M}_{a d}\right) \subset$ $X$ are metrizable (see, e.g., [R, p. 203]). Thus, the above argument shows that $\mathbf{m} \mapsto$ $\mathcal{T}(t) \mathbf{m}$ is continuous with respect to these topologies. Since $\mathcal{C}$ is compact, it maps weakly convergent sequences to strongly convergent sequences, and we have the desired result.

The optimization problem posed is an infinite dimensional one, so we need some approximation methods for compuational purposes. We assume that we have for the original problem a numerical scheme that produces a sequence of semigroups $T^{N}(t ; q)$ defined on finite dimensional subspaces $X^{N}$ of $X$, taken to be of the form $X^{N}=\operatorname{span}\left\{\psi_{1}^{N}, \ldots, \psi_{N}^{N}\right\}$, with $P^{N}$ being the orthogonal projection from $X$ to $X^{N}$. From these semigroups we construct cost functionals

$$
J^{N}(\mathbf{m})=\sum_{k=1}^{n}\left\|\hat{u}\left(t_{k}\right)-\mathcal{C} \mathcal{T}^{N}(t) \mathbf{m}(Q)\right\|_{Z}^{2}
$$

where $\mathcal{T}^{N}$ is defined in the obvious manner. This cost functional must be minimized over a finite dimensional set. Let $\left\{q^{1}, q^{2}, \ldots\right\}$ be a countable, dense subset of $Q$. We set

$$
\mathcal{M}^{N}=\left\{\sum_{j, k=1}^{N} a_{j k} \psi_{j}^{N} \delta_{q^{k}}(d q):\left|\sum_{j, k=1}^{N} a_{j k} \psi_{j}^{N} \delta_{q^{k}}\right|(Q) \leq M\right\}
$$


which is a finite dimensional subset of $\mathcal{M}_{a d}$. Furthermore (as in [F]), any element of $\mathcal{M}_{a d}$ can be approximated in the weak-* sense by a sequence from these sets. With these observations, we may obtain the following approximation result.

Theorem 3.2. Assume that the hypotheses of Theorem 3.2 hold. Furthermore, assume that the sequence $T^{N}(t ; q)$ satisfies $T^{N}(t ; q) P^{N} x \rightarrow T(t ; q) x$, and $\left(T^{N}\right)^{*}(t ; q) P^{N} x \rightarrow$ $T^{*}(t ; q) x$ strongly in $X$ and uniformly on $Q \times[0, \tau]$. Then minimizers of $J^{N}$ over $\mathcal{M}^{N}$ converge subsequentially in the weak-* topology of $\mathcal{M}_{a d}$ to minimizers of $J$.

Proof. In view of the abstract least squares theory of Banks (see, e.g., [BK, pp. 144-5]) it only remains to show that $J^{N}\left(\mathbf{m}^{N}\right) \rightarrow J(\mathbf{m})$, whenever $\mathbf{m}^{N} \rightarrow \mathbf{m}$ in the weak-* sense. Toward that end, we note that

$$
\begin{aligned}
\left\langle x, \int_{Q} T^{N}(t ; q) P^{N} \varphi^{N}(q) \mu^{N}(d q)\right\rangle= & \int_{Q}\left\langle x, T^{N}(t ; q) P^{N} \varphi^{N}(q)\right\rangle \mu^{N}(d q) \\
= & \int_{Q}\left\langle P^{N} x, T^{N}(t ; q) P^{N} \varphi^{N}(q)\right\rangle \mu^{N}(d q) \\
& -\int_{Q}\left\langle x, T(t ; q) \varphi^{N}(q)\right\rangle \mu^{N}(d q) \\
& \quad+\int_{Q}\left\langle x, T(t ; q) \varphi^{N}(q)\right\rangle \mu^{N}(d q) \\
= & \int_{Q}\left\langle\left(T^{N}(t ; q)\right)^{*} P^{N} x, \varphi^{N}(q)\right\rangle \mu^{N}(d q) \\
& -\int_{Q}\left\langle T^{*}(t ; q) x, \varphi^{N}(q)\right\rangle \mu^{N}(d q) \\
& +\left\langle x, \int_{Q} T(t ; q) \varphi^{N}(q) \mu^{N}(d q)\right\rangle \\
\rightarrow & \left\langle x, \int_{Q} T(t ; q) \varphi(q) \mu(d q)\right\rangle,
\end{aligned}
$$

which implies $\mathcal{T}^{N}(t) \mathbf{m}^{N} \rightarrow \mathcal{T}(t) \mathbf{m}$ weakly in $X$. As in Theorem 3.1, we then obtain the desired convergence for $J^{N}$. 


\section{Conclusions.}

We have examined here a semigroup formulation of a rate distribution model for structured populations. Being based merely on an original semigroup formulation for subpopulations of identical individuals, this model is quite general. Using basic properties of vector measures, we studied the basic well-posed question for the semigroup formulation of the distributed model, and developed an estimation framework for fitting the model to data.

We have performed a variety of computational examples based on the Sinko-Streifer problem mentioned above. The implementation involves solving the Sinko-Streifer PDE for many growth rate functions, and we have successfully used the 128-processor Intel iPSC/860 at Oak Ridge National Laboratory for the computations. These results, reported in [BFZ], demonstrate not only the computational feasibility of the techniques we have described, but also the capability of obtaining high quality fits to real observed size structured data that were not previously treatable with inverse techniques.

Future studies will focus on vector measure formulations for nonlinear problems (such as $[\mathrm{H}]$ ) as well as models which incorporate subpopulation mixing (as in [BKW]). 


\section{References.}

[BBKW] Banks, H. T., L. Botsford, F. Kappel, and C. Wang. Modeling and Estimation in Size Structured Population Models, Proceedings $2^{\text {nd }}$ Course on Mathematical Ecology, (Trieste, December 8-12, 1986), World Press, Singapore (1988), 521-541.

[BF] Banks, H. T., and B. G. Fitzpatrick. Estimation of Growth Rate Distributions in Size Structured Population Models, Q. Applied Math, 49, no 2, 1991, pp. 215-235.

[BFZ] Banks, H. T., B. G. Fitzpatrick, and Y. X. Zhang. "A Parallel Algorithm for Rate Distribution Estimation in Size Structured Population Models," to appear.

[BKW] Banks, H. T., F. Kappel, and C. Wang. "Semigroup formulation of a heterogeneous population model and its nonlinear perturbations," to appear.

[BK] Banks, H. T., and K. Kunisch. "Estimation Techniques for Distributed Parameter Systems," Birkhäuser, Boston, 1989.

[DU] Diestel, J., and J. J. Uhl, Jr. "Vector Measures," Mathematical Surveys 15, American Mathematical Society, Providence, 1977.

[D] Dinculeanu, N. "Vector Measures," Permagon Press, Oxford, 1967.

[DS] Dunford, N., and J. T. Schwartz. "Linear Operators, Part I: General Theory," Wiley Interscience, New York, 1966.

[F] Fitzpatrick, B. G. Modeling and Estimation Problems for Structured Heterogeneous Populations, J. Math. Anal. Appl., 172 no. 1, 1993, pp. 73-91.

[H] Huyer, W. "A Size Structured Population Model with Dispersion," J. Math. Anal. Appl., to appear.

[W] Warga, J. "Optimal Control of Differential and Functional Equations," Academic Press, New York, 1972. 